\title{
Reversible cerebral vasoconstriction syndrome without typical thunderclap headache: high- resolution magnetic resonance imaging features
}

\author{
Eun-Hyeok Choi ${ }^{1}$, Inwu Yu ${ }^{1}$, Jae Hong Park ${ }^{1}$, Cindy W. Yoon ${ }^{2}$, Oh Young Bang ${ }^{1}$ \\ ${ }^{1}$ Department of Neurology, Samsung Medical Center, Sungkyunkwan University School of Medicine, Seoul, Korea \\ ${ }^{2}$ Department of Neurology, Inha University School of Medicine, Incheon, Korea
}

Received: December 6, 2018

Revised: December 12, 2018

Accepted: December 14, 2018

Corresponding author:

Inwu Yu

Department of Neurology,

Samsung Medical Center,

Sungkyunkwan University

School of Medicine, 81 Irwon-

ro, Gangnam-gu, Seoul 06351,

Korea

Tel: +82-2-3410-3599

E-mail: i2032@hanmail.net

\begin{abstract}
Reversible cerebral vasoconstriction syndrome (RCVS) is characterized by sudden onset severe headache with or without focal neurologic deficits and is accompanied by segmental or multifocal intracranial arterial vasospasms that resolve within 3 months. The typical RCVS has thunderclap headache but patients with RCVS without this type of headache have been reported. Herein we introduce an unusual case of RCVS without thunderclap headache, together with typical high-resolution magnetic resonance imaging (HR-MRI) features of RCVS showing the possible mechanisms of this condition. The present case suggests that HR-MRI features like dynamic negative remodeling but no enhancement may be a suspicious sign for RCVS, especially in cases with atypical presentation. HR-MRI can be helpful in direct visualization of the vasoconstriction of RCVS and differential diagnosis of other diseases, possibly even without serial examinations. Further research should be performed to test the diagnostic accuracy of HR-MRI in patients with RCVS.
\end{abstract}

Keywords: Cerebral infarction; Headache disorders, primary; High resolution MRI; Reversible cerebral vasoconstriction syndrome

\section{INTRODUCTION}

This is an Open Access article distributed under the terms of the Creative Commons Attribution Non-Commercial License (http:// creativecommons.org/licenses/ by-nc/4.0/).
Reversible cerebral vasoconstriction syndrome (RCVS) is characterized by a sudden onset severe headache with or without focal neurologic deficit, accompanied by segmental or multifocal intracranial arterial vasospasms that resolve within 3 months [1]. Since the feature of RCVS is a reversible cerebral arterial vasospasm, it can cause various brain lesions including subarachnoid hemorrhage, intracerebral hemorrhage, or posterior reversible encephalopathy syndrome, and even ischemic strokes [1,2].

With a recent increase in awareness, RCVS has become the most important cause of thunderclap headache $[3,4]$. It has been demonstrated that thunderclap headache is usually the initial symptom of RCVS. Up to $82 \%$ to $100 \%$ of patients with RCVS have repeated attacks of 
thunderclap headaches during the clinical course $[2,5,6]$. As a result, a headache with thunderclap onset (reaching the peak intensity in less than a minute), of severe intensity, and lasting for 5 minutes or more is considered typical for RCVS [7]. However, atypical presentation of RCVS has been reported. Thus, the diagnosis of RCVS should not be discarded based on the absence of thunderclap headache [8].

Herein we introduce a patient without thunderclap headache who was diagnosed with RCVS based on high-resolution magnetic resonance imaging (HR-MRI) features.

\section{CASE REPORT}

A right-handed 32-year-old previously healthy woman was admitted to the Department of Neurology because of a wakeup-onset right-sided weakness. She had a history of taking a number of unknown oral medications for weight loss from her friends. Several days before stroke onset, she experienced abdominal discomfort and mild headache. Cognitive impairment was reported by her parents and a detailed neurological examination was performed. The clinical examination confirmed her right hemiparesis with hemihypesthesia and revealed acalculia, right-left-disorientation, and finger agnosia which could be termed as Gerstman syndrome.

Brain computed tomography showed no evidence of intra- cranial or extracranial hemorrhage. Brain MRI revealed acute multiple scattered infarctions involving both middle cerebral and anterior cerebral artery territories (Fig. 1A). The largest diffusion restriction was located on the left parietal lobe, which was in agreement with her clinical symptoms. Magnetic resonance (MR) angiography demonstrated multiple focal stenotic segments on the bilateral middle cerebral, the anterior cerebral, and the posterior cerebral arteries. Conventional angiography showed multiple, rather diffuse stenoses, but no basal collaterals (i.e., moyamoya vessels) (Fig. 1B).

Blood count and biochemical analysis results were normal. Further laboratory examinations were performed for differentiation of vasculitis and coagulopathy, including protein C, protein S, anti-thrombin III, anti-phospholipid antibodies, complements, anti-neutrophil cytoplasm antibody, anti-nuclear antibody, anti-double-stranded DNA antibody, anti-RO/ SSA, and anti-La/SSB, but the results were all negative.

Longitudinal transcranial Doppler studies showed dynamic changes after treatment with a calcium channel blocker. The maximal flow velocity of the left and the right middle cerebral arteries was 186 and $210 \mathrm{~cm} / \mathrm{sec}$, respectively. Transcranial Doppler study performed after 1 week of treatment with oral nimodipine $90 \mathrm{mg} /$ day showed reduced maximal flow velocity of the left and the right middle cerebral arteries to 131 and $120 \mathrm{~cm} / \mathrm{sec}$, respectively.
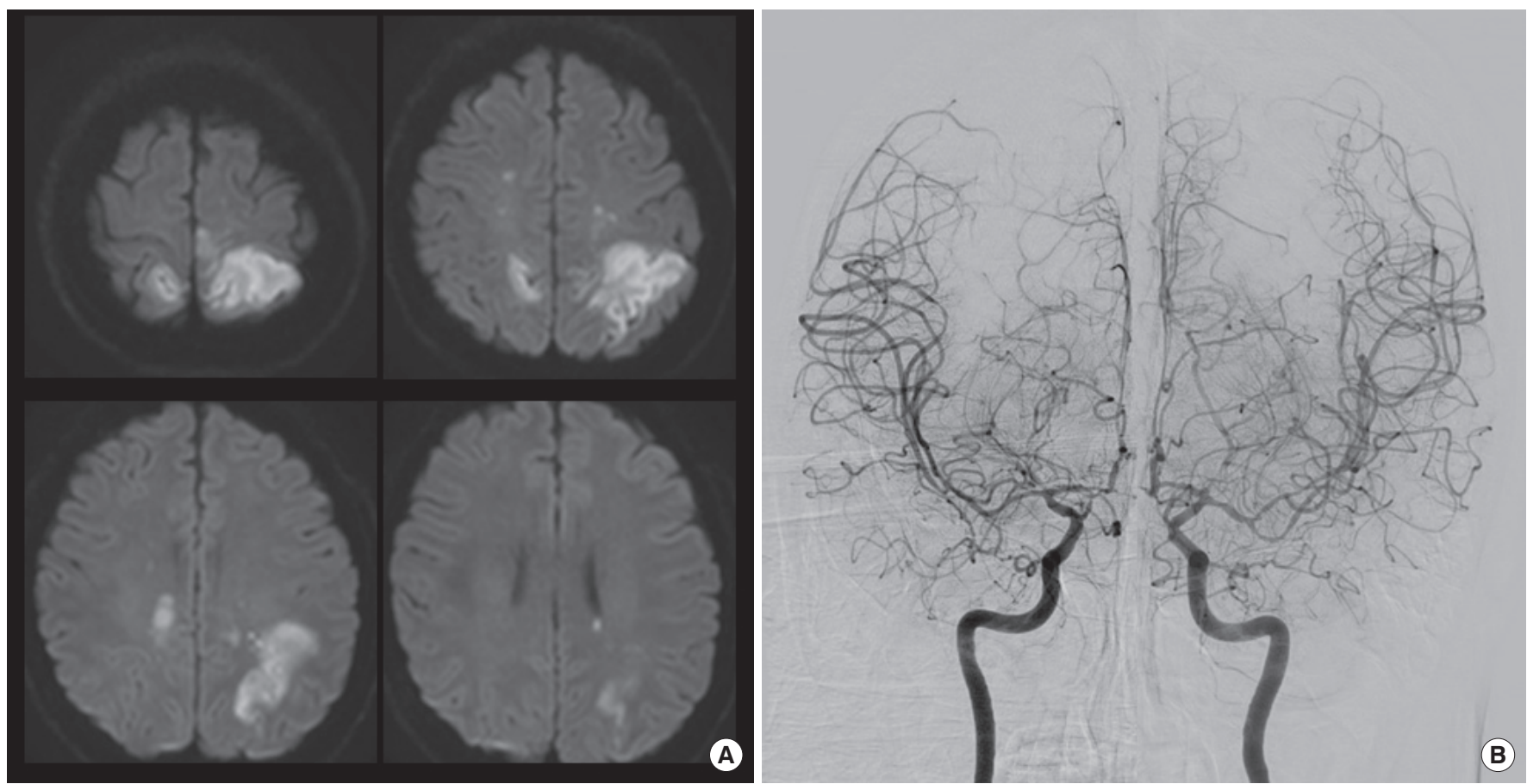

Fig. 1. (A) Magnetic resonance diffusion weighted image revealed acute multiple scattered infarctions involving both middle cerebral and anterior cerebral artery territories. (B) Conventional angiography showed multiple, rather diffuse stenoses, but no basal collaterals. 

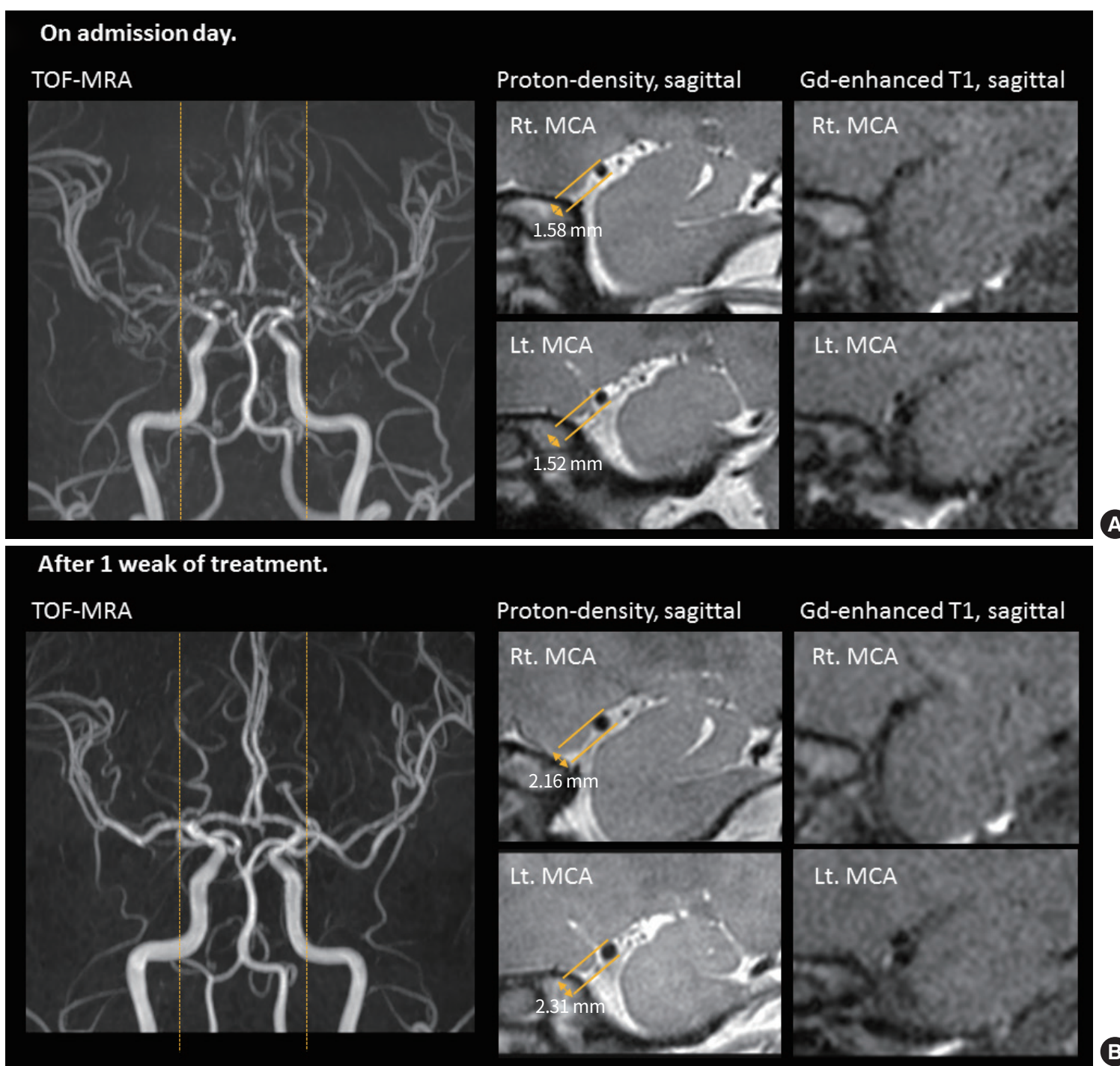

B

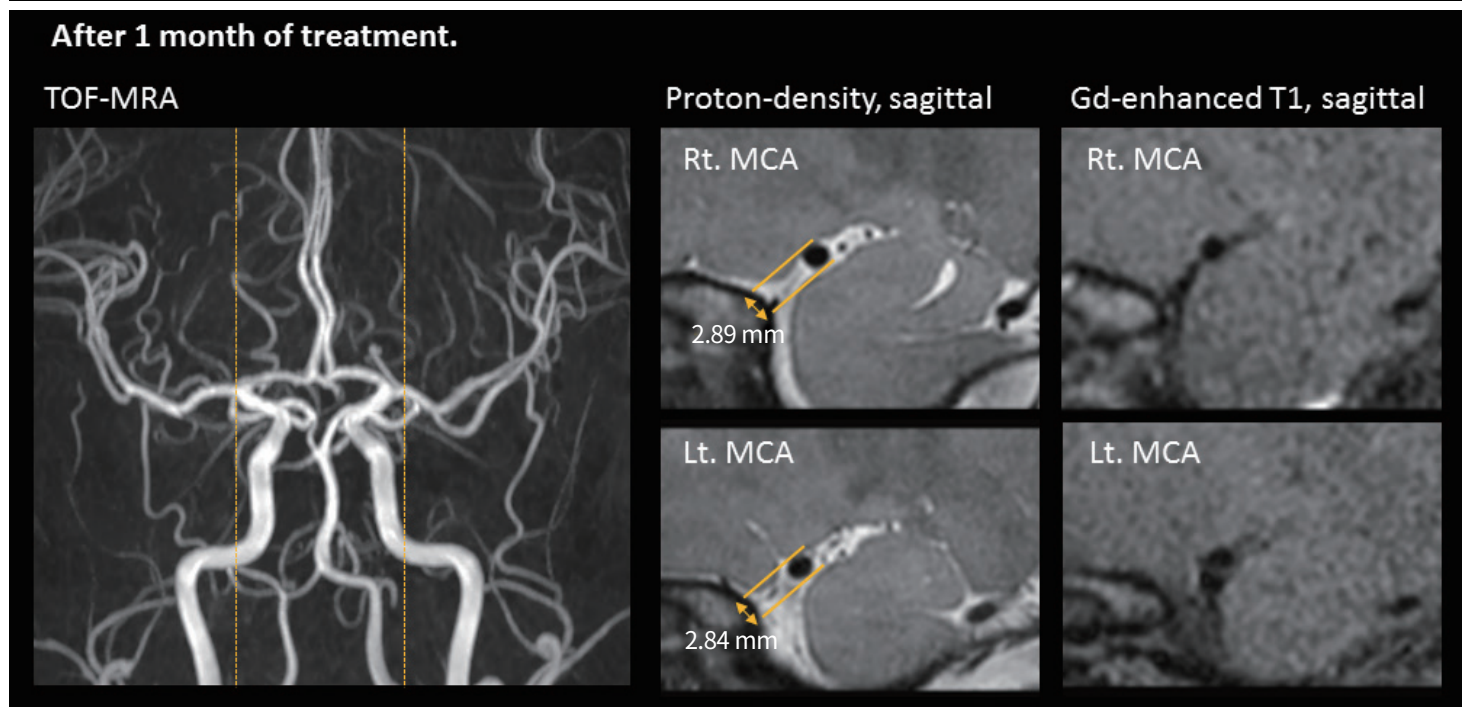

Fig. 2. (A) High-resolution magnetic resonance imaging (HR-MRI) on admission showed diffuse negative remodeling of intracranial arteries without gadolinium enhancement. (B) HR-MRI after 1 week of treatment showed some improvement of the negative remodeling. (C) HR-MRI after 1 month of treatment showed the outer diameter returned to normal values. TOF-MRA, time-of-flight magnetic resonance angiography; Rt., right; MCA, middle cerebral artery; Lt., left. 
HR-MRI was performed longitudinally, on admission, after 1 week, and after 1 month of nimodipine treatment. HR-MRI on admission showed diffuse negative remodeling of intracranial arteries without gadolinium enhancement (Fig. 2A). The outer diameter of the proximal middle cerebral arteries was $1.58 \mathrm{~mm}$ for the right side and $1.52 \mathrm{~mm}$ for the left side. Plaque or pathognomic features of dissection were not present. HR-MRI studies performed after 1 week of treatment with nimodipine showed some improvement of the negative remodeling (Fig. 2B). There was no stenotic segment on time-of-flight MR angiography but the outer diameters of the proximal middle cerebral arteries were still small $(2.16 \mathrm{~mm}$ for the right side and $2.31 \mathrm{~mm}$ for the left side). The outer diameter returned to normal values after 1 month. The outer diameter of the proximal middle cerebral arteries was 2.89 $\mathrm{mm}$ for the right side and $2.84 \mathrm{~mm}$ for the left side (Fig. 2C).

\section{DISCUSSION}

RCVS was first named in 2007 [1] and it has been increasingly recognized in recent years, although it is still an underdiagnosed disease [9]. Considering its potential to cause severe neurological complications, the beneficial effects of calcium channel blockers and possible harmful effects of steroids, early diagnosis would be beneficial for the patients $[7,10]$. However, early diagnosis of RCVS is often challenging. Luminal imaging findings are not specific for RCVS pathophysiology and initial angiography may be normal. The absence of thunderclap headache at onset of other symptoms is exceptional but a literature review identified 87 patients with RCVS without thunderclap headache [8]. Moreover, patients may be aphasic, in an altered mental state, or may be unaware of the headache due to the presence of more severe symptoms [8]. In addition, headache/neck pain can be found in some patients with cervicocephalic dissection and vasculitis, among other diseases.

With the increasing use of longitudinal vascular imaging and HR-MRI, the confirm of disease progress and the number of affected patients are expected to increase in the future. In this context, neuroimaging techniques for differential diagnosis of RCVS from other large intracranial stenotic diseases are needed. Luminal images, including MR angiography, computed tomographic angiography, and conventional angiography are methods of choice for studying RCVS, which reveal cerebral vasoconstrictions and their reversal. Both are essential components for RCVS diagnosis. However, intracranial stenosis can be caused by various diseases such as pri- mary angiitis of the central nerve system (CNS) [11] and RCVS is a high dynamic condition that requires longitudinal imaging studies. Since recently, HR-MRI for vessel wall imaging is being used in patients with RCVS [12-14]. Several case studies suggested that HR-MRI may enable differentiation between RCVS and CNS vasculitis by the absence and presence of arterial wall enhancement $[13,14]$. HR-MRI can also be used for differential diagnosis of other large-vessel intracranial arterial diseases $[11,12,15]$. Intracranial atherosclerosis can be diagnosed by the documentation of atherosclerotic plaques commonly narrowing the lumen (eccentric enhancement if vulnerable) with positive remodeling in most cases. Moyamoya disease can be diagnosed by the negative remodeling and concentric enhancement of symptomatic segments and arterial dissection can be diagnosed by the presence of an intimal flap, double lumen, and mural hematoma. Vasculitis is suspected in cases with concentric enhancement [15-18]. Unlike conditions mentioned above, RCVS does not show pathognomic HR-MRI findings. As shown in the present case, a pathologic process within the vascular wall, i.e., a distinct enhancement or thickening, is usually not present in RCVS. This can help to exclude other disease conditions earlier before the follow-up imaging is obtained. However, it should be mentioned that the pathological process of RCVS first includes distal arteries and then progresses proximally towards the circle of Willis [1], so the diagnostic yield of HR-MRI could be low, especially in the earlier stages of RCVS.

The patient presented here shows longitudinal changes of reversible vasoconstriction. A histopathological study showed that the mean arterial diameter of the middle cerebral artery was $3.13 \mathrm{~mm}$ [19]. In the presented patient, the outer diameter of the middle cerebral artery was 1.52 to $1.58 \mathrm{~mm}$ at the peak of symptoms, which was increased to 2.84 to $2.89 \mathrm{~mm}$ after neurological improvements. Unpredictable and transient failure of regulation of cerebral artery tone with sympathetic overactivity may have a role in the development of RCVS [1]. The differential temporal profile of the early disappearance of headache and the later peak of vasoconstriction causing cerebrovascular events suggest that thunderclap headaches are probably not caused by vasoconstriction [7].

In conclusion, HR-MRI features can provide a diagnostic clue for RCVS, especially in cases with atypical presentation. HR-MRI directly visualizes the vessel walls; thus, it can be helpful for the differential diagnosis of intracranial steno-occlusive diseases. Further research should be performed to test the diagnostic accuracy of HR-MRI in patients with RCVS. 
Written informed consent was obtained from the patient for publication of this case report and any accompanying images.

\section{CONFLICTS OF INTEREST}

No potential conflict of interest relevant to this article was reported.

\section{ACKNOWLEDGMENTS}

This study was supported by a grant from the Ministry of Science and Information and Communication Technology (2018 R1A2B2003489).

\section{REFERENCES}

1. Calabrese LH, Dodick DW, Schwedt TJ, Singhal AB. Narrative review: reversible cerebral vasoconstriction syndromes. Ann Intern Med 2007;146:34-44.

2. Ducros A, Boukobza M, Porcher R, Sarov M, Valade D, Bousser MG. The clinical and radiological spectrum of reversible cerebral vasoconstriction syndrome. A prospective series of 67 patients. Brain 2007;130(Pt 12):3091-101.

3. Chen SP, Wang SJ. Hyperintense vessels: an early MRI marker of reversible cerebral vasoconstriction syndrome? Cephalalgia 2014;34:1038-9.

4. Miller TR, Shivashankar R, Mossa-Basha M, Gandhi D. Reversible cerebral vasoconstriction syndrome, part 1: epidemiology, pathogenesis, and clinical course. AJNR Am J Neuroradiol 2015;36:1392-9.

5. Singhal AB, Hajj-Ali RA, Topcuoglu MA, Fok J, Bena J, Yang $D$, et al. Reversible cerebral vasoconstriction syndromes: analysis of 139 cases. Arch Neurol 2011;68:1005-12.

6. Chen SP, Fuh JL, Lirng JF, Chang FC, Wang SJ. Recurrent primary thunderclap headache and benign CNS angiopathy: spectra of the same disorder? Neurology 2006;67: 2164-9.

7. Ducros A. Reversible cerebral vasoconstriction syndrome. Lancet Neurol 2012;11:906-17.

8. Wolff V, Ducros A. Reversible cerebral vasoconstriction syndrome without typical thunderclap headache. Headache 2016;56:674-87.
9. Chen SP, Fuh JL, Wang SJ. Reversible cerebral vasoconstriction syndrome: an under-recognized clinical emergency. Ther Adv Neurol Disord 2010;3:161-71.

10. Singhal AB, Topcuoglu MA. Glucocorticoid-associated worsening in reversible cerebral vasoconstriction syndrome. Neurology 2017;88:228-36.

11. Bang OY, Toyoda K, Arenillas JF, Liu L, Kim JS. Intracranial large artery disease of non-atherosclerotic origin: recent progress and clinical implications. J Stroke 2018;20:20817.

12. Mossa-Basha M, Hwang WD, De Havenon A, Hippe D, Balu $\mathrm{N}$, Becker KJ, et al. Multicontrast high-resolution vessel wall magnetic resonance imaging and its value in differentiating intracranial vasculopathic processes. Stroke 2015;46:1567-73.

13. Mandell DM, Matouk CC, Farb RI, Krings T, Agid R, terBrugge $\mathrm{K}$, et al. Vessel wall MRI to differentiate between reversible cerebral vasoconstriction syndrome and central nervous system vasculitis: preliminary results. Stroke 2012;43:860-2.

14. Obusez EC, Hui F, Hajj-Ali RA, Cerejo R, Calabrese LH, Hammad T, et al. High-resolution MRI vessel wall imaging: spatial and temporal patterns of reversible cerebral vasoconstriction syndrome and central nervous system vasculitis. AJNR Am J Neuroradiol 2014;35:1527-32.

15. Lehman VT, Brinjikji W, Kallmes DF, Huston JR, Lanzino G, Rabinstein AA, et al. Clinical interpretation of high-resolution vessel wall MRI of intracranial arterial diseases. $\mathrm{Br} J$ Radiol 2016;89:20160496.

16. Ryoo S, Lee MJ, Cha J, Jeon P, Bang OY. Differential vascular pathophysiologic types of intracranial atherosclerotic stroke: a high-resolution wall magnetic resonance imaging study. Stroke 2015;46:2815-21.

17. Ryoo S, Cha J, Kim SJ, Choi JW, Ki CS, Kim KH, et al. High-resolution magnetic resonance wall imaging findings of Moyamoya disease. Stroke 2014;45:2457-60.

18. Park MS, Cha J, Chung JW, Seo WK, Kim GM, Bang OY. Arterial dissection as a cause of intracranial stenosis in East Asians. J Am Coll Cardiol 2017;70:2205-6.

19. Yang WJ, Wong KS, Chen XY. Intracranial atherosclerosis: from microscopy to high-resolution magnetic resonance imaging. J Stroke 2017;19:249-60. 